PROCEEDINGS OF THE

AMERICAN MATHEMATICAL SOCIETY

Volume 132, Number 1, Pages 37-46

S 0002-9939(03)07033-3

Article electronically published on May 9, 2003

\title{
FREE PRODUCTS IN LINEAR GROUPS
}

\author{
D. S. PASSMAN
}

(Communicated by Lance W. Small)

\begin{abstract}
Let $R$ be a commutative integral domain of characteristic 0 , and let $G$ be a finite subgroup of $\operatorname{PGL}_{n}(R)$, the projective general linear group of degree $n$ over $R$. In this note, we show that if $n \geq 2$, then $\operatorname{PGL}_{n}(R)$ also contains the free product $G * T$, where $T$ is the infinite cyclic group generated by the image of a suitable transvection.
\end{abstract}

\section{INTRODUCTION}

Let $R$ be a commutative integral domain of characteristic 0 , and let $\operatorname{GL}_{n}(R)$ denote the general linear group of degree $n$ over $R$, namely the group of invertible $n \times n R$-matrices. If $R^{\bullet}$ is the set of scalar matrices in $\mathrm{GL}_{n}(R)$, then $R^{\bullet}$ is isomorphic to the group of units of $R$, and $\mathrm{GL}_{n}(R) / R^{\bullet}=\mathrm{PGL}_{n}(R)$ is the projective general linear group. Our goal here is to show that if $G$ is a finite subgroup of $\operatorname{PGL}_{n}(R)$ and if $n \geq 2$, then $\mathrm{PGL}_{n}(R)$ also contains the free product $G * T$, where $T$ is the infinite cyclic group generated by the image of a suitable transvection, namely a transformation of the form $1+\tau$, where $\tau$ has rank 1 and square 0 .

The above proposition actually arose as part of an argument to show that if $H$ is a finite group having a noncentral subgroup $G$ of prime order $p$, then the unit group of the integral group ring $Z[H]$ contains the free product $G * T$ for some infinite cyclic group $T$. Obviously, the proof of such a result must use the irreducible representations of the rational group algebra $Q[H]$ and then, under suitable conditions, properties of linear groups over characteristic 0 integral domains. Since the linear group results turned out to be of independent interest, they are being published separately. Indeed, a second paper [GM], written at the same time as this work, contains an alternate approach to the existence of free products in linear groups.

For the most part, we work in $\mathrm{GL}_{n}(C)$, where $C$ is the field of complex numbers, and the key result here is

Theorem 1.1. Let $V$ be a finite-dimensional complex vector space, and let $G$ be a subgroup of the general linear group $\mathrm{GL}(V)$ with $\left|G:\left(G \cap C^{\bullet}\right)\right|<\infty$. Furthermore, let $\tau: V \rightarrow V$ be a nonzero linear transformation of square 0 , and write $K=\operatorname{ker} \tau$ and $I=\operatorname{im} \tau=\tau(V)$. If $g I \cap K=0$ for all $g \in G \backslash\left(G \cap C^{\bullet}\right)$, then for all sufficiently large complex numbers $c \in C$, we have

$$
\langle G, 1+c \tau\rangle /\left(G \cap C^{\bullet}\right) \cong\left(G /\left(G \cap C^{\bullet}\right)\right) * T,
$$

Received by the editors August 26, 2002.

2000 Mathematics Subject Classification. Primary 20E06, 20H20.

(C)2003 American Mathematical Society 
where $T$ is the infinite cyclic group generated by the image of the unit $1+c \tau$ in this factor group.

Observe that $(1+c \tau)(1-c \tau)=1-c^{2} \tau^{2}=1$, so $1+c \tau \in \operatorname{GL}(V)$ and, of course, $\langle G, 1+c \tau\rangle$ indicates the subgroup of $\mathrm{GL}(V)$ generated by $G$ and the element $1+c \tau$. As will be apparent, the proof of Theorem 1.1 contains enough information to compute the lower bound on the size of $c$ when $\tau$ has rank 1 . Indeed, we have

Corollary 1.2. Let $V$ be a finite-dimensional complex inner product vector space, let $G$ be a subgroup of the general linear group $\operatorname{GL}(V)$ with $\left|G:\left(G \cap C^{\bullet}\right)\right|<\infty$, and assume that $\left(C^{\bullet} G\right) \cap \mathrm{SL}(V)$ acts in a unitary manner on $V$. Furthermore, let $\tau: V \rightarrow V$ be a linear transformation of square 0 and rank 1 , write $I=\operatorname{im} \tau=C v$, $K=\operatorname{ker} \tau$, and suppose that $g v \notin K$ for all $g \in G \backslash\left(G \cap C^{\bullet}\right)$. If $m$ is the minimum value of $|\tau(g v)| /|g v|$ over all $g \in G \backslash\left(G \cap C^{\bullet}\right)$, and if $c$ is a complex number with $|c| \geq 27\|\tau\| / m^{2}$, then we have

$$
\langle G, 1+c \tau\rangle /\left(G \cap C^{\bullet}\right) \cong\left(G /\left(G \cap C^{\bullet}\right)\right) * T,
$$

where $T$ is the infinite cyclic group generated by the image of the unit $1+c \tau$ in this factor group.

Presumably the factor 27 above can be appreciably decreased with more care, but we will not pursue this further. Note that, if $A$ and $B$ are groups, then the free product $A * B$ contains the free product of the conjugate subgroups $A^{b}=b^{-1} A b$ for all $b \in B$. In particular, the preceding results have a number of obvious corollaries. Less obvious is

Theorem 1.3. Let $R$ be a characteristic 0 integral domain and let $G$ be a finite subgroup of $\mathrm{PGL}_{n}(R)$. If $n \geq 2$, then $\mathrm{PGL}_{n}(R)$ contains the free product $G * T$, where $T$ is the infinite cyclic group generated by the image of a suitable transvection $1+\tau \in \mathrm{SL}_{n}(Z) \subseteq \mathrm{SL}_{n}(R)$.

As an immediate consequence, we obtain

Corollary 1.4. Let $R$ be a characteristic 0 integral domain and let $G$ be a finite subgroup of $\mathrm{GL}_{n}(R)$ with $G \cap R^{\bullet}=1$. If $n \geq 2$, then $\mathrm{GL}_{n}(R)$ contains the free product $G * T$, where $T$ is the infinite cyclic group generated by a suitable transvection $1+\tau \in \mathrm{SL}_{n}(Z) \subseteq \mathrm{SL}_{n}(R)$.

As is to be expected, the proof of Theorem 1.1 ultimately depends upon the "ping-pong" lemma of F. Klein (see [H, Lemma II.24]). For convenience, we state and quickly prove this result in precisely the form we require.

Lemma 1.5. Let $\Gamma$ be a group generated by subgroups $G$ and $T$, and let $G$ contain a normal subgroup $\Delta$ of $\Gamma$. Suppose $\Gamma$ acts on a set $X$ and let $P$ and $Q$ be disjoint nonempty subsets of $X$. If $\Delta Q \subseteq Q,(G \backslash \Delta) P \subseteq Q,(T \backslash 1) Q \subseteq P$, and $|T|>2$, then $\Gamma / \Delta \cong(G / \Delta) * T$.

Proof. It suffices to show that no element $\gamma \in \Delta$ can be written as a nonempty alternating product of elements coming from $G \backslash \Delta$ and $T \backslash 1$. Suppose by way of contradiction that such a product $\gamma=\gamma_{1} \gamma_{2} \cdots \gamma_{n}$ exists with $n \geq 1$. If the product starts and ends in $G \backslash \Delta$, that is, if $\gamma_{1}, \gamma_{n} \in G \backslash \Delta$, then by conjugating this expression by a nonidentity element of $T$, we obtain a similar expression, but this time starting and ending in $T \backslash 1$. Next, if $\gamma_{1} \in G \backslash \Delta$ and $\gamma_{n} \in T \backslash 1$, then since $|T|>2$, we can conjugate $\gamma$ by an element of $T \backslash\left\{1, \gamma_{n}^{-1}\right\}$ to obtain a similar 
product but starting and ending in $T \backslash 1$. Since the same argument handles the $\gamma_{1} \in T \backslash 1, \gamma_{n} \in G \backslash \Delta$ situation, we can therefore replace any such expression by one with $\gamma_{1}, \gamma_{n} \in T \backslash 1$. But then, the alternating nature of the action of $G \backslash \Delta$ and $T \backslash 1$ on $P$ and $Q$ yields $\gamma Q \subseteq Q$ and $\gamma_{1} \gamma_{2} \cdots \gamma_{n} Q \subseteq P$, and this is a contradiction since $\gamma=\gamma_{1} \gamma_{2} \cdots \gamma_{n}$, and since $P$ and $Q$ are disjoint nonempty subsets of $X$.

\section{Proof of Theorem 1.1}

The goal of this section is to prove Theorem 1.1 and its corollary. To start with, we suppose that $V$ is a finite-dimensional complex vector space and that $G$ is a subgroup of $\operatorname{GL}(V)$ with $\left|G:\left(G \cap C^{\bullet}\right)\right|<\infty$. If $H=C^{\bullet} G \cap \mathrm{SL}(V)$, then it is clear that $G \subseteq C^{\bullet} H$ and that $\left|H:\left(H \cap C^{\bullet}\right)\right|<\infty$. Furthermore, since $H \cap C^{\bullet}$ consists of scalar operators of determinant 1 , it follows that $\left|H \cap C^{\bullet}\right| \leq \operatorname{dim}_{C} V<\infty$. Thus $H$ is a finite group and, as is well known, there exists a Hermitian inner product ( , ) defined on $V$ with $H$ acting as unitary transformations. Indeed, if [, ] is any Hermitian inner product, then we merely define $(a, b)=\sum_{h \in H}[h a, h b]$ for all $a, b \in V$. Now fix any such inner product ( , ), let $S=\{v \in V \mid(v, v)=1\}$ be the unit sphere in $V$, and define the real-valued distance function $d: V^{\bullet} \times V^{\bullet} \rightarrow R$ by

$$
d(a, b)=\left|\frac{a}{|a|}-\frac{b}{|b|}\right| \geq 0
$$

for all nonzero vectors $a, b \in V$. Since $d(a, b) \leq|a /| a||+|b /| b||=2$, we see that $V$ has $d$-diameter at most 2 . Indeed, the diameter is precisely 2 since $d(a,-a)=2$.

Lemma 2.1. Let $0 \neq a, b \in V$.

(i) If $\lambda \in C^{\bullet}$, then $d(\lambda a, \lambda b)=d(a, b)$.

(ii) If $g \in G$, then $d(g a, g b)=d(a, b)$.

(iii) $d(a, b) \leq 2|a-b| /|a|$.

Proof. Part (i) is clear and then (ii) follows since $G \subseteq C^{\bullet} H$ and since $H$ consists of unitary transformations. For (iii), note that

$$
v=\frac{a}{|a|}-\frac{b}{|b|}=\frac{a-b}{|a|}-\frac{b}{|b|} \cdot \frac{|a|-|b|}{|a|}=v^{\prime}-v^{\prime \prime} .
$$

Since $\left|v^{\prime}\right|=|a-b| /|a|$ and $\left|v^{\prime \prime}\right|=|| a|-| b|| /|a| \leq|a-b| /|a|$, it follows that

$$
d(a, b)=|v| \leq\left|v^{\prime}\right|+\left|v^{\prime \prime}\right| \leq \frac{2|a-b|}{|a|},
$$

as required.

Now let $A$ and $B$ be subsets of $V$ with $A^{\bullet}, B^{\bullet} \neq \emptyset$, where $A^{\bullet}=A \backslash 0$. Then we define

$$
d(A, B)=d\left(A^{\bullet}, B^{\bullet}\right)=\inf \left\{d(a, b) \mid a \in A^{\bullet}, b \in B^{\bullet}\right\} .
$$

We are particularly interested in subsets of $V$ closed under multiplication by $C^{\bullet}$. Since these correspond (except for a possible 0 element) to subsets of the projective space of $V$, we call these projective subsets of $V$. Observe that if $A$ is projective, then $\left\{a /|a| \mid a \in A^{\bullet}\right\}=A \cap S$ and $A^{\bullet}=C^{\bullet}(A \cap S)$, where the latter is the set of all products, not sums of products. Hence, for every subset $B \subseteq V$, we have $d(A, B)=$ $d(A \cap S, B)$. In particular, if $B$ is also projective, then $d(A, B)=d(A \cap S, B \cap S)$. 
Lemma 2.2. Let $A, B \subseteq V$ with $A^{\bullet}, B^{\bullet} \neq \emptyset$.

(i) If $\lambda \in C^{\bullet}$, then $d(\lambda A, \lambda B)=d(A, B)$. In particular, if $B$ is projective, then $d(\lambda A, B)=d(A, B)$, so $d\left(C^{\bullet} A, B\right)=d(A, B)$.

(ii) If $g \in G$, then $d(g A, g B)=d(A, B)$.

(iii) If $A$ and $B$ are subspaces of $V$, then $d(A, B)=\left|a_{0}-b_{0}\right|$ for some $a_{0} \in A \cap S$, $b_{0} \in B \cap S$. In particular, if $A \cap B=0$, then $d(A, B)>0$.

Proof. Parts (i) and (ii) are immediate from the corresponding parts of Lemma 2.1. For (iii), we note that $A$ and $B$ are projective sets, so

$$
d(A, B)=d(A \cap S, B \cap S)=\inf \{|a-b| \mid a \in A \cap S, b \in B \cap S\} .
$$

Thus the result follows since $A \cap S$ and $B \cap S$ are compact and since ||$: V \rightarrow R$ is a continuous function.

We now turn to the

Proof of Theorem 1.1. Recall that $I=\operatorname{im} \tau, K=\operatorname{ker} \tau$, and $g I \cap K=0$ for all $g \in G \backslash\left(G \cap C^{\bullet}\right)$. We use the inner product and distance function as given above, and we proceed in a series of steps.

Step 1. Notation and the definitions of $\varepsilon, P$ and $Q$.

Proof. If $g \in G \backslash\left(G \cap C^{\bullet}\right)$, then $g I \cap K=0$, so $d(g I, K)>0$ by Lemma 2.2(iii). Thus since $C^{\bullet} I=I$ and $\left|G:\left(G \cap C^{\bullet}\right)\right|<\infty$, we can choose a real number $\varepsilon>0$ so that $d(g I, K) \geq 3 \varepsilon$ for all elements $g \in G$ not contained in $G \cap C^{\bullet}$. Note that $\varepsilon \leq 2 / 3$ since $V$ has diameter 2 . Let

$$
P=\left\{v \in V^{\bullet} \mid d(v, I)<\varepsilon\right\} .
$$

Then $P \supseteq I^{\bullet}$, so $P \neq \emptyset$. Furthermore, since $I$ is a projective set, it follows from Lemma 2.2(i) that $P$ is also a projective set.

If $g \in G$, then Lemma 2.2(ii) implies that

$$
g P=\left\{g v \in V^{\bullet} \mid d(v, I)<\varepsilon\right\}=\left\{w \in V^{\bullet} \mid d(w, g I)<\varepsilon\right\},
$$

and we define

$$
Q=\bigcup_{g \in G \backslash(G \cap C \bullet)} g P
$$

Then $Q \neq \emptyset$ and, by definition, we have $\left(G \backslash\left(G \cap C^{\bullet}\right)\right) P \subseteq Q$. Note also that $P$ and $Q$ are projective sets, so $\left(G \cap C^{\bullet}\right) P \subseteq P$ and $\left(G \cap C^{\bullet}\right) Q \subseteq Q$.

Step 2. $d(K, Q) \geq 2 \varepsilon$ and hence $P \cap Q=\emptyset$.

Proof. We use the fact that $K, Q$ and $I$ are all projective sets. Let $a \in K \cap S$ and $b \in Q \cap S$. Then $b \in g P \cap S$ for some $g \in G \backslash\left(G \cap C^{\bullet}\right)$, so the definition of $g P$ implies that there exists $c \in g I \cap S$ with $|b-c|=d(b, c)<\varepsilon$. Now $|a-c|=$ $d(a, c) \geq d(K, g I) \geq 3 \varepsilon$, so

$$
|a-b|+\varepsilon>|a-b|+|b-c| \geq|a-c| \geq 3 \varepsilon,
$$

and therefore $|a-b|>2 \varepsilon$. Since $d(K, Q)$ is the infimum of these values $|a-b|$, we conclude that $d(K, Q) \geq 2 \varepsilon$.

Finally, $\tau^{2}=0$, so $I \subseteq K$ and hence $d(I, Q) \geq d(K, Q) \geq 2 \varepsilon$. In particular, if $v \in Q$, then $d(v, I) \geq d(Q, I) \geq 2 \varepsilon>\varepsilon$. Thus $v \notin P$, and hence $P \cap Q=\emptyset$. 
Step 3. There exists a real number $r>0$ with the property that if $\lambda \in C$ with $|\lambda| \geq r$, then $(1+\lambda \tau) Q \subseteq P$.

Proof. Write $V=K \dot{+} Y$, a direct sum of subspaces. Since $K=\operatorname{ker} \tau$ and $I=\operatorname{im} \tau$, the restriction of $\tau$ to $Y$ yields an invertible linear transformation $\sigma: Y \rightarrow I$. Thus $\sigma^{-1}: I \rightarrow Y$ and we let $s^{-1}=\left\|\sigma^{-1}\right\|$ be the norm of this map. In other words, $\left|\sigma^{-1} z\right| \leq s^{-1}|z|$ for all $z \in I$. In particular, if $y \in Y$, then $y=\sigma^{-1}(\tau y)$, so $|y| \leq s^{-1}|\tau y|$ and $|\tau y| \geq s|y|$. Set $r=3 /\left(s \varepsilon^{2}\right)$ and note that $r s \varepsilon-1=(3-\varepsilon) / \varepsilon>2 / \varepsilon$ since $1>\varepsilon>0$.

Now let $v \in Q^{\bullet}$, so $d(v, K) \geq d(Q, K) \geq 2 \varepsilon$ by Step 2 , and write $v=x+y \in$ $K \dot{+} Y=V$ with $x \in K$ and $y \in Y$. If $x=0$, then $v=y$, so $|y|=|v| \geq \varepsilon|v|$ since $1>\varepsilon>0$. On the other hand, if $x \neq 0$, then, by Lemma 2.1(iii) with $a=v$ and $b=x$, we have $2|y| /|v| \geq d(v, x) \geq d(Q, K) \geq 2 \varepsilon$, so again we obtain $|y| \geq \varepsilon|v|$. In other words, $|y| \geq \varepsilon|v|$ in all cases and hence $y \neq 0$. Now let $\lambda \in C$ with $|\lambda| \geq r$, and note that $1+\lambda \tau$ is an invertible linear transformation on $V$ with inverse $1-\lambda \tau$. Thus $w=(1+\lambda \tau) v \neq 0$.

Since $x \in K=\operatorname{ker} \tau$, we have

$$
w=(1+\lambda \tau) v=v+\lambda \tau v=v+\lambda \tau(x+y)=v+\lambda \tau y
$$

and $\lambda \tau y \in I$. Furthermore, $y \neq 0$, so $\tau y=\sigma y \neq 0$ and hence $\lambda \tau y \neq 0$. Thus, by Lemma 2.1(iii) again with $a=w$ and $b=\lambda \tau y$, we have

$$
d(w, I) \leq d(w, \lambda \tau y) \leq 2|v| /|w| .
$$

Now $|\lambda \tau y|=|\lambda||\tau y| \geq|\lambda| s|y| \geq|\lambda| s \varepsilon|v|$, so

$$
|w|=|v+\lambda \tau y| \geq|\lambda \tau y|-|v| \geq(|\lambda| s \varepsilon-1)|v| .
$$

Indeed, since $|\lambda| \geq r$, we have $|\lambda| s \varepsilon-1 \geq r s \varepsilon-1>2 / \varepsilon$, and hence $|w|>2|v| / \varepsilon$. Consequently, $d(w, I) \leq 2|v| /|w|<\varepsilon$ and $w \in P$, as required.

Step 4. Completion of the proof.

Proof. Let $c \in C$ with $|c| \geq r$, let $t=1+c \tau$ and write $T=\langle t\rangle$. Since $t^{n}=1+n c \tau$, we see that $t^{n}=1$ if and only if $n=0$, and hence $T$ is infinite cyclic. Furthermore, if $n \neq 0$, then $|n c| \geq|c| \geq r$, so Step 3 implies that $t^{n} Q \subseteq P$. In other words, $(T \backslash 1) Q \subseteq P$. We also observed in Steps 1 and 2 that $\left(G \backslash\left(G \cap C^{\bullet}\right)\right) P \subseteq Q$ and that $P \cap Q=\emptyset$. In particular, since $|T|>2$, we conclude from Lemma 1.5 that $\langle G, T\rangle /\left(G \cap C^{\bullet}\right) \cong\left(G /\left(G \cap C^{\bullet}\right)\right) * T$, and the theorem is proved.

Next we show that the proof of Theorem 1.1 contains enough information to compute specific bounds when $\tau$ has rank 1. For this, we first indicate how to compute the distance between a nonzero vector and a subspace of the vector space. Again, we assume that $V$ is a finite-dimensional complex vector space having a Hermitian inner product $($,$) .$

Lemma 2.3. Let $0 \neq v \in V$ and let $A$ be a nonzero subspace of $V$.

(i) If $(v, A)=0$, then $d(v, A)^{2}=2$.

(ii) If $(v, A) \neq 0$, write $B=A \cap v^{\perp}$, so that $B$ is a subspace of $A$ of codimension 1 , and let $A=C a \dot{+} B$, where $C a=A \cap B^{\perp}$. Then

$$
d(v, A)^{2}=2\left(1-\frac{|(v, a)|}{|v||a|}\right) .
$$


Proof. We can assume that $|v|=1$.

(i) If $(v, A)=0$ and $x \in A \cap S$, then $d(v, x)^{2}=|v-x|^{2}=|v|^{2}+|x|^{2}=2$ since $v$ and $x$ are perpendicular.

(ii) We can clearly assume that $|a|=1$. If $x \in A$ with $|x|=1$, then $x=\lambda a+b$ with $\lambda \in C, b \in B$ and with $1=|x|^{2}=|\lambda|^{2}+|b|^{2}$. Next, we have

$$
\begin{aligned}
d(v, x)^{2} & =|v-x|^{2}=|v|^{2}+|x|^{2}-(v, x)-(x, v) \\
& =2-(v, \lambda a)-(\lambda a, v)=2-2 \Re \mathrm{e}(\bar{\lambda}(v, a)) .
\end{aligned}
$$

This is clearly minimized when $|\lambda|=1$ and when $\bar{\lambda}(v, a)$ is real and positive. Thus $\lambda=(v, a) /|(v, a)|$ and $d(v, A)^{2}=2-2|(v, a)|$.

With this, we can prove

Lemma 2.4. Let $v, \alpha \in V \backslash 0$ and let $\tau: V \rightarrow V$ be the linear transformation given by $\tau(x)=(x, \alpha) v$ for all $x \in V$.

(i) If $K=\operatorname{ker} \tau$ and $0 \neq w \in V$, then $d(w, K) \geq|(w, \alpha)| /(|w||\alpha|)$.

(ii) $\|\tau\|=|\alpha||v|$.

Proof. Since $\tau(x)=(x, \alpha /|\alpha|)|\alpha| v$, it suffices to assume that $|\alpha|=1$. Furthermore, for part (i), we may assume that $|w|=1$.

(i) If $(w, K)=0$, then $d(w, K)=\sqrt{2}>|w||\alpha| \geq|(w, \alpha)|$ by the first part of the previous lemma. Thus we can suppose that $(w, K) \neq 0$, and we use the notation of the second part above. Since $K=\alpha^{\perp}$, we see that $B=\alpha^{\perp} \cap w^{\perp}$, and note that $a=w-(w, \alpha) \alpha \in K$. Moreover, $(a, K)=(w, K) \neq 0$, so $a \neq 0$, and it is clear that $a \perp B$. Thus Lemma 2.3(ii) implies that $d(w, K)^{2}=2-2|(w, a)| /|a|$. Now

$$
(w, a)=(w, w)-|(w, \alpha)|^{2}=1-|(w, \alpha)|^{2},
$$

and

$$
|a|^{2}=|w|^{2}+|(w, \alpha)|^{2}-2|(w, \alpha)|^{2}=1-|(w, \alpha)|^{2} .
$$

It therefore follows that

$$
d(w, K)^{2}=2-2 \sqrt{1-|(w, \alpha)|^{2}} \geq|(w, \alpha)|^{2},
$$

so $d(w, K) \geq|(w, \alpha)|$, as required.

(ii) Observe that $V=C \alpha \dot{+} K$ is an orthogonal direct sum of subspaces. In particular, if $x \in V \backslash K$, then $x=\lambda \alpha+k$ for some $0 \neq \lambda \in C$ and $k \in K$, and hence $|x|^{2}=|\lambda|^{2}|\alpha|^{2}+|k|^{2}=|\lambda|^{2}+|k|^{2}$. Furthermore, $\tau(x)=\tau(\lambda \alpha)=\lambda(\alpha, \alpha) v=\lambda v$. Thus

$$
\frac{|\tau(x)|}{|x|}=\frac{|\lambda||v|}{\sqrt{|\lambda|^{2}+|k|^{2}}}=\frac{|v|}{\sqrt{1+|k|^{2} /|\lambda|^{2}}} \leq|v|,
$$

and it is clear that $\|\tau\|=|v|$.

We close this section with the

Proof of Corollary 1.2. We follow the proof of Theorem 1.1 and use its notation. Furthermore, since $\tau: V \rightarrow V$ has rank 1 with $0 \neq v \in I=\operatorname{im} \tau$, we can assume that $\tau(x)=(x, \alpha) v$ for some fixed vector $0 \neq \alpha \in V$. Of course, $K=\operatorname{ker} \tau=\alpha^{\perp}$.

Note that, by Lemma $2.2(\mathrm{i})$, we have $d(g I, K)=d(C g v, K)=d(g v, K)$ for any $g \in G$. Furthermore, by Lemma 2.4(i), we have

$$
d(g v, K) \geq|(g v, \alpha)| /(|g v||\alpha|)=|\tau(g v)| /(|g v||v||\alpha|) .
$$


Thus, by definition, we can take $\varepsilon$ to be

$$
\varepsilon=\frac{1}{3} \min \left\{\frac{|\tau(g v)|}{|g v||v||\alpha|} \mid g \in G \backslash\left(G \cap C^{\bullet}\right)\right\}=\frac{m}{3|v||\alpha|},
$$

where $m$ is the minimum value of $|\tau(g v)| /|g v|$ over all $g \in G \backslash\left(G \cap C^{\bullet}\right)$.

Next, observe that $V=K \dot{+} C \alpha$ and that $\tau$ restricted to $C \alpha$ determines an isomorphism $\sigma: C \alpha \rightarrow I$ given by $\lambda \alpha \mapsto \lambda|\alpha|^{2} v$. Thus $\sigma^{-1}: \mu v \mapsto \mu \alpha /|\alpha|^{2}$ for all $\mu \in C$ and hence $s^{-1}=\left\|\sigma^{-1}\right\|=1 /(|v||\alpha|)$. Finally, we set

$$
r=\frac{3}{s \varepsilon^{2}}=\frac{27|v||\alpha|}{m^{2}}=\frac{27\|\tau\|}{m^{2}}
$$

by Lemma 2.4(ii). But $r$ is the lower bound for the size of the complex numbers $c$ given by the proof of Theorem 1.1, so the result follows.

\section{Proof of Theorem 1.3}

In this section, we quickly prove Theorem 1.3 and its corollary. Then we discuss two examples of interest. We start with

Proposition 3.1. Let $R$ be a subring of the complex numbers $C$ and let $G$ be a subgroup of $\mathrm{GL}_{n}(R)$ with $\left|G:\left(G \cap R^{\bullet}\right)\right|<\infty$. If $n \geq 2$, then there exists a transvection $1+\tau \in \mathrm{SL}_{n}(Z) \subseteq \mathrm{SL}_{n}(R)$ such that

$$
\langle G, 1+t \tau\rangle /\left(G \cap R^{\bullet}\right) \cong\left(G /\left(G \cap R^{\bullet}\right)\right) *\langle 1+t \tau\rangle
$$

for all sufficiently large $t \in R$ (measured in $C$ ).

Proof. Of course, $\mathrm{GL}_{n}(R) \subseteq \mathrm{GL}_{n}(C)$, and we let $\mathrm{GL}_{n}(C)$ act on the $C$-vector space $V \cong C^{n}$. Furthermore, let $V^{\prime} \cong Q^{n}$ embed naturally in $V$, where $Q$ is the field of rational numbers. For each $g \in G \backslash\left(G \cap R^{\bullet}\right)$, the eigenspaces for $g$ in $V$, with eigenvalues in $C$, are finitely many proper subspaces of $V$. Moreover, it is clear that all group elements in the coset $g\left(G \cap R^{\bullet}\right)$ have the same eigenspaces, but with possibly different eigenvalues. Thus since $\left|G:\left(G \cap R^{\bullet}\right)\right|<\infty$, the eigenspaces for all elements $g \in G \backslash\left(G \cap R^{\bullet}\right)$ constitute just finitely many proper subspaces of $V$, say these are $V_{1}, V_{2}, \ldots, V_{k}$. In particular, since $C V^{\prime}=V$, the intersections $V_{i}^{\prime}=V_{i} \cap V^{\prime}$ are finitely many proper $Q$-subspaces of $V^{\prime}$. Thus, since $Q$ is an infinite field, we have $\bigcup_{i=1}^{k} V_{i}^{\prime} \neq V^{\prime}$, and hence we can choose $v \in V^{\prime}$ not in any of these proper subspaces. It follows that $g v \notin C v$ for all $g \in G \backslash\left(G \cap R^{\bullet}\right)$. Indeed, since $g\left(G \cap R^{\bullet}\right) C v=C g v$, we obtain just finitely many 1-dimensional subspaces of $V$ in this manner, and they are all distinct from $C v$.

Note that the images of these lines in $V / C v$ determine finitely many subspaces $L_{1} / C v, L_{2} / C v, \ldots, L_{\ell} / C v$, with $\operatorname{dim}_{C} L_{i}=2$. Now consider the vector space dual $W$ of $V$, and let $W^{\prime}=\left\{\lambda \in W \mid \lambda\left(V^{\prime}\right) \subseteq Q\right\}$ be the rational subspace naturally embedded in $W$. If $\widetilde{W}=\{\lambda \in W \mid \lambda(C v)=0\}$, then each $\widetilde{W}_{i}=\left\{\lambda \in W \mid \lambda\left(L_{i}\right)=\right.$ $0\}$ is a proper subspace of $\widetilde{W}$, so it follows easily as above that there exists a linear functional $f \in W^{\prime}$ with $f(C v)=0$, but with $f(C g v) \neq 0$ for all $g \in G \backslash\left(G \cap R^{\bullet}\right)$. Now define $\tau: V \rightarrow V$ by $\tau(x)=f(x) v$ for all $x \in V$. Obviously, $\tau$ has rank 1 with image $I=C v$ and with $\operatorname{ker} \tau=\operatorname{ker} f=K$ of codimension 1 in $V$. Furthermore, by the definitions of $V^{\prime}$ and $W^{\prime}, \tau$ corresponds to a matrix with entries in $Q$. In particular, we can multiply $\tau$ by a nonzero element of $Z$ to clear denominators. This new $\tau$ corresponds to a $Z$-matrix, but with the same image $I$ and kernel $K$ and, since $f(v)=0$, we have $I \subseteq K$ and hence $\tau^{2}=0$. On the other hand, since 
$f(g v) \neq 0$ and since $I$ is 1-dimensional, we have $g I \cap K=C g v \cap K=0$ for all $g \in G \backslash\left(G \cap R^{\bullet}\right)$, and therefore Theorem 1.1 yields the result.

Note that, in the above argument, if $V_{i}^{\prime}=V_{i} \cap V^{\prime} \neq 0$ and if $V_{i}$ is an eigenspace for $g \in G \subseteq \mathrm{GL}_{n}(R)$, then the corresponding eigenvalue is certainly contained in $F$, the field of fractions of $R$. With Proposition 3.1 in hand, Theorem 1.3 is now essentially obvious. There is just one small observation that needs to be made.

Proof of Theorem 1.3. Since $G$ is finite, there exists a finitely generated subgroup $H$ of $\mathrm{GL}_{n}(R)$ such that $H /\left(H \cap R^{\bullet}\right)=G$. We can now assume that $R$ is generated by the finitely many entries in the matrices representing these finitely many generators of $H$ and in the matrices of their inverses. In other words, $R$ is a countable characteristic 0 domain, and hence it can be embedded in the complex numbers $C$. By Proposition 3.1, there exists a transvection $1+\tau \in \mathrm{SL}_{n}(Z) \subseteq \mathrm{SL}_{n}(R)$ such that

$$
\langle H, 1+t \tau\rangle /\left(H \cap R^{\bullet}\right) \cong\left(H /\left(H \cap R^{\bullet}\right)\right) *\langle 1+t \tau\rangle=G *\langle 1+t \tau\rangle
$$

for all sufficiently large $t \in R$. Furthermore, note that $\langle H, 1+t \tau\rangle \cap R^{\bullet}=H \cap R^{\bullet}$. Indeed, this is obvious if $G=1$, and it is immediate when $G \neq 1$ since $G *\langle 1+t \tau\rangle$ has trivial center. This completes the proof.

In a real sense, the final argument above using the center of the free product is unnecessary. A close look at the proof of Theorem 1.1 shows that the ping-pong lemma is applied to the action of the group $\langle G, 1+c \tau\rangle$ on certain projective subsets of $V$. Furthermore, the proof of that lemma not only shows that $\langle G, 1+c \tau\rangle /\left(G \cap C^{\bullet}\right)$ is a free product, but also that it acts faithfully when permuting the sets $P$ and $Q$. As a consequence, $\langle G, 1+c \tau\rangle /\left(G \cap C^{\bullet}\right)$ acts faithfully on the projective space of $V$.

Next, we have

Proof of Corollary 1.4. Let ${ }^{-}: \mathrm{GL}_{n}(R) \rightarrow \mathrm{PGL}_{n}(R)$ be the natural map. Since $\bar{G}$ is finite, Theorem 1.3 implies that there exists a transvection $1+\tau \in \mathrm{SL}_{n}(Z) \subseteq \mathrm{SL}_{n}(R)$ such that $\langle\bar{G}, \bar{T}\rangle \cong \bar{G} * \bar{T}$, where $T=\langle 1+\tau\rangle$. But $\bar{G} \cong G$ and $\bar{T} \cong T$, so we have $\langle G, T\rangle \cong G * T$, as required.

We close this paper by considering two examples of interest. The first one comes from GM.

Example 3.2. Let $V$ be a complex vector space with basis $\left\{v_{0}, v_{1}, \ldots, v_{n}\right\}$, and let $G \subseteq \mathrm{GL}(V)$ be a subgroup of order $n+1$ that regularly permutes the basis vectors. Suppose $\tau: V \rightarrow V$ is defined by $\tau\left(v_{i}\right)=a_{i} v_{0}$ with $a_{0}=0$, but with $0 \neq a_{i} \in C$ for all $i=1,2, \ldots, n$. Then $\langle G, 1+c \tau\rangle=G *\langle 1+c \tau\rangle$ for all complex numbers $c$ that satisfy

$$
|c| \geq \frac{27\left(\left|a_{1}\right|^{2}+\left|a_{2}\right|^{2}+\cdots+\left|a_{n}\right|^{2}\right)^{1 / 2}}{\min \left\{\left|a_{1}\right|^{2},\left|a_{2}\right|^{2}, \ldots,\left|a_{n}\right|^{2}\right\}} .
$$

Proof. Let the Hermitian inner product (, ) be defined on $V$ so that $\left\{v_{0}, v_{1}, \ldots, v_{n}\right\}$ is an orthonormal basis. Then certainly $G$ acts as unitary operators on $V$. Furthermore, if we set $\alpha=\bar{a}_{1} v_{1}+\bar{a}_{2} v_{2}+\cdots+\bar{a}_{n} v_{n}$, then $\tau(x)=(x, \alpha) v_{0}$ for all $x \in V$. Since $G \cap C^{\bullet}=1$, Corollary 1.2 implies that $\langle G, 1+c \tau\rangle=G *\langle 1+c \tau\rangle$ if $c$ is a complex number with $|c| \geq 27\|\tau\| / m^{2}$, where $m=\min \left\{\left|\tau\left(g v_{0}\right)\right| /\left|g v_{0}\right| \mid g \in G \backslash 1\right\}$. By Lemma 2.4(ii), $\|\tau\|=|\alpha|\left|v_{0}\right|=\left(\left|a_{1}\right|^{2}+\left|a_{2}\right|^{2}+\cdots+\left|a_{n}\right|^{2}\right)^{1 / 2}$. Furthermore, if $g \in G \backslash 1$, then $g v_{0}=v_{i}$ for some $i \neq 0$, and all such $v_{i}$ occur. Thus, since 
$\tau\left(g v_{0}\right)=\tau\left(v_{i}\right)=a_{i} v_{0}$, it follows that $m=\min \left\{\left|a_{i}\right| \mid i \neq 0\right\}$ and, in particular, we have $m^{2}=\min \left\{\left|a_{i}\right|^{2} \mid i \neq 0\right\}$.

In a recent unpublished note, Dan Goldstein showed that if $p$ is an odd prime and $\zeta$ is a primitive complex $p$ th root of unity, then $\mathrm{SL}_{2}\left(Q\left[\zeta+\zeta^{-1}\right]\right)$ contains the free product $G_{1} * G_{2}$ of two cyclic groups of order $p$. Our final example is motivated by this result.

Example 3.3. Let $p$ be an odd prime and let $\zeta$ be a complex primitive $p$ th root of unity. Let

$$
g=\left(\begin{array}{cc}
0 & 1 \\
-1 & \zeta+\zeta^{-1}
\end{array}\right) \quad \text { and } \quad h=\left(\begin{array}{cc}
-1 & 1 \\
-1 & 1
\end{array}\right)
$$

be $2 \times 2$ matrices over the ring $R=Z\left[\zeta+\zeta^{-1}\right]$. If $r \in R$ with $|r| \geq p^{3} / 2$, then $\mathrm{SL}_{2}(R)$ contains the free product $G * T$, where $G=\langle g\rangle$ is cyclic of order $p$ and $T=\langle 1+r h\rangle$ is infinite cyclic.

Proof. We work in the larger $\operatorname{ring} Z[\zeta]$. If

$$
\ell=\left(\begin{array}{cc}
1 & 1 \\
\zeta^{-1} & \zeta
\end{array}\right) \text {, then } \ell^{-1} g \ell=\left(\begin{array}{cc}
\zeta^{-1} & 0 \\
0 & \zeta
\end{array}\right) \text { and } \ell^{-1} h \ell=\mu\left(\begin{array}{cc}
1 & -\zeta \\
\zeta^{-1} & -1
\end{array}\right)
$$

where $\mu=(1-\zeta) /(1+\zeta)$. Thus, it suffices to assume that $V$ has basis $\left\{v_{1}, v_{2}\right\}$ with $g v_{1}=\zeta^{-1} v_{1}$ and $g v_{2}=\zeta v_{2}$. Furthermore, if $v=\zeta^{1 / 2} v_{1}+\zeta^{-1 / 2} v_{2}$, then $h=\mu \tau$ where $\tau\left(v_{1}\right)=v_{1}+\zeta^{-1} v_{2}=\zeta^{-1 / 2} v$ and $\tau\left(v_{2}\right)=-\left(\zeta v_{1}+v_{2}\right)=-\zeta^{1 / 2} v$. Now assume that $($,$) is an inner product on V$ with $v_{1}$ and $v_{2}$ orthonormal vectors. Then $g$ is a unitary operator on $V$ and $\tau(x)=(x, \alpha) v$, where $\alpha=\zeta^{1 / 2} v_{1}-\zeta^{-1 / 2} v_{2}$. By Lemma 2.4(ii), $\|\tau\|=|\alpha||v|=2$, and $\tau\left(g^{i} v\right)=\left(g^{i} v, \alpha\right) v=\left(\zeta^{-i}-\zeta^{i}\right) v$. Thus $\left|\tau\left(g^{i} v\right)\right| /\left|g^{i} v\right|=\left|\zeta^{i}-\zeta^{-i}\right|=\left|2 \Im \mathrm{m}\left(\zeta^{i}\right)\right|$, and hence

$$
m=\min \left\{\frac{\left|\tau\left(g^{i} v\right)\right|}{\left|g^{i} v\right|} \mid i=1,2, \ldots, p-1\right\}=2 \sin (\pi / p) .
$$

By Corollary 1.2, if $c$ is a complex number with $|c| \geq 27\|\tau\| / m^{2}=27 /\left(2 \sin ^{2}(\pi / p)\right)$, then $\langle G, 1+c \tau\rangle \cong G *\langle 1+c \tau\rangle$.

In particular, if $r \in R$ with $|r \mu| \geq 27 /\left(2 \sin ^{2}(\pi / p)\right)$, then $\langle G, 1+r h\rangle \cong G *\langle 1+r h\rangle$. Finally, observe that $\mu=(1-\zeta) /(1+\zeta)=\left(\zeta^{-1 / 2}-\zeta^{1 / 2}\right) /\left(\zeta^{-1 / 2}+\zeta^{1 / 2}\right)$, so $|\mu|=$ $\left|\Im \mathrm{m}\left(\zeta^{1 / 2}\right)\right| /\left|\Re \mathrm{e}\left(\zeta^{1 / 2}\right)\right|$. In particular, the smallest value for $|\mu|$ over all embeddings of $Z[\zeta]$ in $C$ is $\tan (\pi / p)$, and hence we need $|r| \geq(27 \cos (\pi / p)) /\left(2 \sin ^{3}(\pi / p)\right)$. Since the latter trigonometric expression is easily seen to be smaller than $p^{3} / 2$, the condition $|r| \geq p^{3} / 2$ guarantees that $\langle G, 1+r h\rangle$ is a free product.

Note that no nonidentity element of the subgroup $G=\langle g\rangle \subseteq \mathrm{SL}_{2}(R)$ can have an eigenvalue in $F=Q\left[\zeta+\zeta^{-1}\right]$. Thus, in view of the proof of Proposition 3.1 and the remarks that follow it, we could take $h$ in the above example to be any nonzero $Z$-matrix of square 0 . For instance, we could take $h$ to be either of the matrix units $e_{1,2}$ or $e_{2,1}$. Of course, the bound on $r$ would necessarily change. Our choice of the particular $h$ in Example 3.3 is therefore somewhat random, but it does seem to be more symmetrically placed with respect to the matrix $g$. Furthermore, the same $h$ can be used for $p=2$ if we take $g=\operatorname{diag}(1,-1) \in \mathrm{GL}_{2}(Z)$.

\section{ACKNOWLEDGEMENT}

The author thanks Dan Goldstein for his helpful comments. 


\section{REFERENCES}

[GM] J. Z. Gonçalves and A. Mandel, Free groups generated by transvections, to appear.

$[\mathrm{H}]$ P. de la Harpe, Topics in Geometric Group Theory, Chicago Lectures in Mathematics, Univ. of Chicago Press, Chicago, 2000. MR 2001i:20081

Department of Mathematics, University of Wisconsin, Madison, Wisconsin 53706

E-mail address: passman@math.wisc.edu 